\title{
Bioimaging Probes for Reactive Oxygen Species and Reactive Nitrogen Species
}

\author{
Tetsuo Nagano ${ }^{1,2, *}$ \\ ${ }^{1}$ Graduate School of Pharmaceutical Sciences and Chemical Biology Research Initiative, The University of Tokyo, \\ 7-3-1, Hongo, Bunkyo-ku, Tokyo 113-0033, Japan \\ ${ }^{2}$ CREST, Japan Science and Technology Agency, 4-8-1, Honcho, Kawaguchi, Saitama 332-0012, Japan
}

Received 20 June, 2009; Accepted 30 June, 2009

\begin{abstract}
Summary Reactive oxygen species (ROS) play key roles in many pathogenic processes, including carcinogenesis, inflammation, ischemia-reperfusion injury and signal transduction. Also, reactive nitrogen species (RNS) cause various biological events such as neurodegenerative disorders. Sensitive and specific detection methods for ROS and RNS in biological samples should be useful for elucidation of biological events both in vitro and in vivo. Fluorescent probes based on small organic molecules have become indispensable tools in modern biology because they provide dynamic information concerning the localization and quantity of biological molecules of interest, without the need of genetic engineering of the sample. In this review, we recount some recent achievements in the field of small molecular fluorescent probes. First, the probes for nitric oxide and peroxynitrite as RNS are introduced and the probes of hydroxyl radical, hydrogen peroxide, hypochlorous and singlet oxygen as ROS are discussed, based on the fluorescence off/on switching mechanisms including photoinduced electron transfer and spirocyclization processes, and with some applications for in vitro and in vivo systems.
\end{abstract}

Key Words: bioimaging probe, reactive oxygen species, reactive nitrogen species, fluorescence, photoinduced electron transfer

\section{Introduction}

For all aerobic organisms including humans, molecular oxygen $\left(\mathrm{O}_{2}\right)$ is essential for survival, serving as the final

\footnotetext{
*To whom correspondence should be addressed.

Tel: +81-3-5841-4850 Fax: +81-3-5841-4855

E-mail: tlong@mol.f.u-tokyo.ac.jp

He received "SFRR Japan Award" in 2008 in recognition of his outstanding work.

\section{Biography}

Dr. Nagano is a full professor at Department of Chemistry and Biology, Graduate School of Pharmaceutical Sciences, the University of Tokyo and also a director general of Chemical Biology Research Initiative at University of Tokyo. Recently, his main
}

electron acceptor of mitochondrial electron transport, and a vital process for synthesizing ATP from ADP. Partially reduced and highly reactive metabolites of $\mathrm{O}_{2}$ may be formed during this or other electron transfer reactions. These metabolites comprise superoxide anion $\left(\mathrm{O}_{2}{ }^{-}\right)$, hydrogen

research interests are in the molecular-design, synthesis and applications of novel bio-imaging probes for physiologically active species such as nitric oxide, $\mathrm{Zn}^{2+}$, singlet oxygen, reactive oxygen, caspase, etc. Until now, he has developed more than 40 bioimaging fluorescent probes according to the rational molecular designs, based on the mechanisms of photoinduced electron transfer (PeT), fluorescence resonance energy transfer (FRET) and intramolecular charge transfer (ICT). Twelve probes among the developed probes are now commercially available and widely used among many biologists in the world. He was awarded Uehara Prize in 2004, Shimadzu Prize in 2005, the Pharmaceutical Society of Japan Award in 2006 and National Medal with Purple Ribon in 2006. 
peroxide $\left(\mathrm{H}_{2} \mathrm{O}_{2}\right)$, and hydroxyl radical ( $\left.\cdot \mathrm{OH}\right)$. These metabolites, together with other oxidizing agents such as $\mathrm{HOCl}$ and singlet oxygen $\left({ }^{1} \mathrm{O}_{2}\right)$, are referred to as "reactive oxygen species (ROS)". Since ROS could potentially damage lipids, proteins and DNA, cells developed several defense mechanisms, which include antioxidant enzymes and targeted degradation pathways. Thus, "oxidative stress" may be defined as an imbalance between ROS production and the antioxidant capacity of the cell. Accumulated evidence suggests that oxidative stress is involved in a variety of human diseases, such as cancer and in aging. Recently, more interests are focused on physiological functions of reactive nitrogen species (RNS) such as nitric oxide (NO) as a ubiquitous messenger molecule in the cardiovascular, nervous and immune systems, and peroxynitrite $\left(\mathrm{ONOO}^{-}\right)$as a species inducing nitrative stress. Thus, specific and sensitive methods to detect the production of ROS and RNS precisely should be required to evaluate their functions in living body.

It is desirable that seeing into the body and cells is accomplished by using less-invansive techniques, without cutting into the body or isolating of cellular constituents. Therefore, techniques to visualize physiological or pathophysiological changes in the body and cells become increasingly important in biomedical sciences.

Compared to other technologies such as radioisotope labeling, MRI, ESR and electrochemical detection, fluorescence imaging has many advantages for this purpose, as it enables highly sensitive, less-invasive and safe detection using readily available instruments. Another advantage of fluorescence imaging we should emphasize here is that the fluorescence signal of a molecule can be drastically modulated, so that probes relying on 'activation', not just accumulation, can be utilized. Fluorescent probes based on small organic molecules have become indispensable tools in modern biology because they provide dynamic information concerning the localization and quantity of the molecules of interest, without the need of genetic engineering of the sample. Until the 1980s, however, fluorescence imaging was mainly applied to fixed samples owing to the lack of fluorescent chemosensors or probes, suitable for imaging in living cells. In this review, "fluorescent probes" are defined as molecules that react specifically with ROS and RNS to induce a concomitant change of their photochemical properties (fluorescence intensity, excitation/emission wavelength, and so forth). Today, several design strategies for fluorescent probes, including photoinduced electron transfer $(\mathrm{PeT})$, fluorescence resonance energy transfer (FRET), intramolecular charge transfer (ICT), and spirocyclization, are well established and have been applied to many probes. Some of them were developed in our laboratory.

Thus, biofunctional species-reactive fluorescent probes which allow bioimaging with high spatial and temporal resolution in conjunction with fluorescence microscopy are useful for elucidation of biological functions. For instance, experimental studies on $\mathrm{Ca}^{2+}$-dependent signal transduction in cells were greatly facilitated by the development of fluorescent probes for $\mathrm{Ca}^{2+}$.

This review introduces fluorescent probes for ROS and RNS bioimaging, and most of these probes are applicable to cellular or in vivo imaging.

\section{Probes for Nitric Oxide}

In 1987 [1] it was reported that NO is identical with endothelium-derived relaxing factor (EDRF), which is biosynthesized in the living body. It is now widely known that NO is a small uncharged free radical containing one unpaired electron, and an ubiquitous messenger molecule in the cardiovascular, nervous, and immune systems. In addition, 1-arginine-derived NO has been found in a wide variety of organisms ranging from mammals to invertebrates, bacteria, and plants.

$\mathrm{NO}$ is biosynthesized by three distinct mammalian NO synthase isoforms. Neuronal NOS (nNOS or NOS I) and endothelial NOS (eNOS or NOS III) are constitutively expressed in neuronal and endothelial cells, respectively, and thus, both enzymes are also referred to as cNOS, while inducible NOS (iNOS or NOS II) is expressed in cells involved in inflammation such as macrophages and microglias through stimulation with cytokines and/or endotoxins. Although cNOS and iNOS do not show a significant difference in specific activity, cNOS activity is $\mathrm{Ca}^{2+}$-dependent whereas iNOS activity is $\mathrm{Ca}^{2+}$-independent, and these NOSs are expressed at different levels from each other. Since the enzyme activity of cNOS is dependent on transiently raised concentrations of intracellular $\mathrm{Ca}^{2+}$, the NO levels produced in stimulated endothelial and neuronal cells are low (submicromolar at the cellular level) relative to those $(1-10 \mu \mathrm{M})$ in macrophages; in addition, the formation rate of $\mathrm{NO}$ in the former cells is higher than in the case of NO generated by other NOS. Thus, cNOS is a transiently activated and lowoutput isoform while iNOS expression results in a sustained production and high-output of NO. Moreover, excessive production of NO has been realized to be responsible for various pathophysiolgies, while far lower levels of NO are produced under physiological conditions.

The most popular reaction of NO in oxygen-containing biological fluids can be an oxidation reaction with $\mathrm{O}_{2}$. The autoxidation of $\mathrm{NO}$ and the subsequent reactions occur rapidly within the membrane. Reported values for the halflife of NO under physiological conditions range from 0.1 to $5 \mathrm{~s}$. The half-life in whole blood is shortened to millisecond order owing to the rapid reaction of NO with oxyhemoglobins. Although NO is a relatively stable free radical, it readily and rapidly reacts with free radicals and metalcontaining proteins in biological systems, exerting physio- 
logical or pathophysiological effects, and therefore, in situ NO detection is required to elucidate the biological roles of NO. In vivo and in situ visualization of NO using bioimaging techniques should provide us with a comprehensive understanding of the production and diffusion processes of NO.

To develop useful fluorescent NO probes, the following criteria should be satisfied: (1) Fluorescent probes should react with NO or NO-derived species to yield the corresponding fluorescent products without forming byproducts at room temperature at atmospheric pressure under physiological pH conditions in an aqueous solution; (2) The reaction should be specific for NO or NO-derived species; (3) Both of the probes and the products should be stable, especially to light, because laser microscopy is used for bioimaging; (4) The fluorescence properties of the probes should be different in the presence and in the absence of NO; (5) Visible light is desirable for excitation to diminish autofluorescence and photodamage to living systems; (6) The probes should be membrane-permeable so that they can enter living cells for bioimaging. In addition, ROS such as $\mathrm{O}_{2}{ }^{--}$, $\mathrm{OH}$, etc., are often generated concomitantly with formation of NO. Thus, a practical detection method is required which is satisfactory for studies in living cells in terms of distinguishability of NO from ROS.

The use of NO-reactive fluorescent probes, in conjunction with confocal laser microscopy, should provide an NO bioimaging technique which is suitable for real-time analysis of intracellular NO. They can be applied to imaging in vivo in whole animal, although some surgical operations might be required to expose the dye-loaded region to the light.

\section{Diaminofluoresceins: $D A F s$}

In attempts to measure NO generated under physiological conditions, we developed a fluorometric method that exploits the ability of NO to produce N-nitrosating agents. The method is based on the use of an aromatic diamino compound (DAN). The relatively nonfluorescent DAN reacts rapidly with $\mathrm{N}$-nitrosating agent(s) to yield the highly fluorescent product (NAT). We found DAN can react with $\mathrm{NO}$ to yield NAT in the presence of $\mathrm{O}_{2}$ under a neutral condition (Fig. 1) [2]. Until then, DAN had been considered as a reagent for the detection of $\mathrm{NO}_{2}{ }^{-}$under acidic conditions. However, the results obtained mean that the reaction of NO with DAN aerobically produces the fluorescent compound NAT quantitatively in an aqueous medium, even
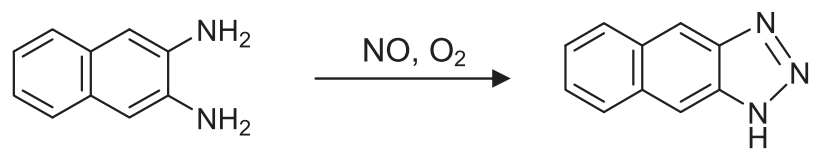

Fig. 1. Reaction of DAN with NO to form NAT in the presence of $\mathrm{O}_{2}$ in neutral buffer under physiological conditions. In fact, the fluorescence intensity increases dose-dependently on addition of NO to the DAN solution. Under these neutral reaction conditions, $\mathrm{NO}_{2}{ }^{-}$and $\mathrm{NO}_{3}{ }^{-}$cannot convert DAN into NAT, which the selectivity for $\mathrm{NO}$ is high.

However, it is impossible to use DAN itself as a probe for the imaging of NO because DAN leaks easily through cell membranes after loading. In addition, the use of DAN presents some problems such as cytotoxicity and strong autofluorescence owing to the requirement for UV excitation, the small extinction coefficient, and the poor solubility in neutral buffer. Therefore, new fluorescent indicators were synthesized to detect NO in living cells as a means to examine the physiological functions of NO.

Fluorescein is widely used in biology as a fluorophore because of its convenient excitation and emission wavelengths for biological measurement, high extinction coefficient, and high fluorescence quantum yield in water. Diaminofluoresceins (DAFs) were therefore designed as novel probes for NO [3-5]. Conversion of DAFs to the corresponding triazole forms (DAF-Ts) by reaction with NO caused little change of the absorbance maxima but greatly increased the quantum efficiency. DAFs possess favorable properties for cellular imaging applications; in particular, they have a visible excitation wavelength, which is less damaging to cells and is not subject to interference from the autofluorescence of biological samples. The increase of fluorescence intensity is dependent on the concentration of NO. The detection limit of NO by DAF-2 is $5 \mathrm{nM}$. The fluorescence of DAF-2 T strikingly decreases below $\mathrm{pH} 7$. That is, DAF-2 is useful in media above $\mathrm{pH}$ 7. DAF-4 and DAF-5 with the electron-withdrawing chlorine substituent were synthesized in order to lower the pKa value, with the aim of obtaining stable fluorescence intensity at physiological pH (Fig. 2). The excitation and emission wavelengths of DAF-4 and DAF-5 with a chlorine atom adjacent to phenolic $\mathrm{OH}$ are shifted to longer wavelengths than that of DAF-2.

On the other hand, fluorinated fluorescein derivatives are reported to be more resistant to photobleaching than fluorescein and can be efficiently excited with the $488 \mathrm{~nm}$ spectral line of the argon-ion laser used in confocal laser scanning microscopes. Therefore, the chlorine atom was replaced with a fluorine atom. This new indicator was named DAF-FM (Fig. 2). Examination of the photobleaching in sunlight showed that the triazole form of DAF-FM (DAFFM T) is more stable than DAF-2 T and DAF-4 T. The fluorescence intensity is stable above $\mathrm{pH}$ 5.8. The sensitivity of DAF-FM is 1.4 times higher than that of DAF-2.

We examined NO bioimaging in cultured bovine aortic endothelial cells using DAF-FM DA [6]. The cultured bovine aortic endothelial cells were incubated with DAFFM DA for $1 \mathrm{~h}$ for dye loading. After stimulation with 
<smiles>[R]c1cc2c(-c3cc(N)c(N)cc3C(=O)O)c3cc([R])c(=O)cc-3oc2cc1O</smiles>

DAF- $2: \mathrm{R}=\mathrm{H}$

DAF-5 : $\mathrm{R}=\mathrm{Cl}$<smiles>CC(=O)Oc1cc2c(cc1F)C1(OC(=O)c3cc(OC(C)=O)c(F)cc31)c1cc(N)c(N)cc1O2</smiles>

DAF-FM DA<smiles>Nc1ccc(-c2c3cc(Cl)c(=O)cc-3oc3cc(O)c(Cl)cc23)c(C(=O)O)c1N</smiles><smiles>O=C(O)c1cc2nn[nH]c2cc1-c1c2ccc(=O)cc-2oc2cc(O)ccc12</smiles><smiles>CNc1ccc(-c2c3cc(F)c(=O)cc-3oc3cc(O)c(F)cc23)cc1N</smiles>

DAF-FM<smiles>Cn1nnc2cc(-c3c4cc(F)c(=O)cc-4oc4cc(O)c(F)cc34)ccc21</smiles>

DAF-FM T

Fig. 2. Structures of DAF compounds

bradykinin, the fluorescence intensity in the cells increased and that in the cytosol increased more than that in the nucleus. NO is produced in the cytosol, where NOS exists. This observation implies that little of the produced NO diffuses into the nucleus. The augmentation of the fluorescence intensity was suppressed by an NOS inhibitor. In conclusion, DAF-FM is a useful tool for visualizing the temporal and spatial distribution of intracellular NO.

DAF-FM DA was also applied to imaging of $\mathrm{NO}$ generated in rat hippocampal slices by exposure to an aglycemic medium [7]. NO production was observed mainly in the CA1 area and was dependent on the concentration of $\mathrm{O}_{2}$. During exposure to an anoxic - aglycemic medium, NO was hardly produced while marked elevation of intracellular $\mathrm{Ca}^{2+}$ was observed. Production of NO increased sharply as soon as the perfusate was changed to the normal medium. These results suggest that NOS is activated after reperfusion rather than during ischemia.

\section{Diaminorhodamines: DARs}

Although the practical usefulness of DAFs for functional studies of NO was confirmed, DAFs lack some desirable characteristics of NO indicators. To obtain higher photostability, longer excitation wavelength and applicability over a wider $\mathrm{pH}$ range, we examined the suitability of rhodamine as the fluorophore.

DARs were designed, based on the highly fluorescent Rhodamine B fluorophore and synthesized from phthalic anhydride derivatives and N,N-diethyl-3-aminophenol. The reaction of DARs and NO affords the corresponding fluorescent triazole, as in the case of DAFs. To image NO in living

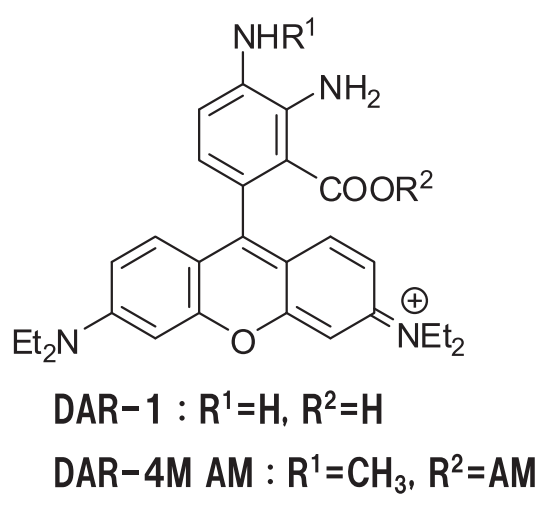

Fig. 3. Structures of DAR compounds

cells, DARs should be membrane-permeable. Therefore, the acetoxymethyl ester of DAR-1 was prepared (DAR-1 AM) because acetoxymethyl esters are easily hydrolyzed by intracellular esterases (Fig. 3). The fluorescence intensity of the triazole form of DAR-4 was stable above $\mathrm{pH} 4$, while that of DAR-1 was unstable at around $\mathrm{pH} 7$.

For bioimaging applications, an acetoxymethyl ester was introduced into DAR-4M (Fig. 3). We then examined the suitability of DAR-4M AM for bioimaging. The quantum efficiency of DAR-4M $\mathrm{T}$ is the highest of all DARs examined. Judging from NO standard curves calibrated with the indicators, the sensitivity of DAR-4M to NO is twice that of DAR-1. The detection limit of NO by DAR-4M was $7 \mathrm{nM}$. DAR-4M should offer a good signal-to-noise ratio in the examination of biological samples because of the low background fluorescence with the longer wavelength 
excitation, even though the quantum yield of DAR-4M T is lower than that of fluorescein probes. Moreover, DAR-4M permitted NO detection above $\mathrm{pH}$, while DAF-FM, a fluorescein probe, can only be applied above $\mathrm{pH} 5.8$.

In addition, DAR-4M AM was applied to the imaging of NO in bovine aortic endothelial cells [8]. DARs are more photostable than DAFs. The fluorescence in cells was observed after stimulation with bradykinin, which raises the intracellular $\mathrm{Ca}^{2+}$ level and thereby activates NOS, and this increase was suppressed by addition of an NOS inhibitor. DAR-4M should be useful for bioimaging of samples that have strong autofluorescence in the case of $490 \mathrm{~nm}$ excitation in which the intracellular $\mathrm{pH}$ may fall below 6 .

\section{Dichlorodiaminocalcein: DCl-DA Cal}

We next designed and synthesized novel fluorescence probes containing the iminodiacetic acid group (IAG). They showed superior intracellular retention, making it possible to visualize low concentrations of target molecules that would be difficult to observe with conventional probes, and permitting long-term observation in living cells. Improvement of intracellular retention of fluorescence probes holds great promise as a strategy for developing a wide range of highly sensitive probes for studies on various biological phenomena.

DAFs are excellent as fluorescence probes for $\mathrm{NO}$; however, their sensitivity is sometimes insufficient to measure NO in living cells. Hence, we designed and synthesized dichlorodiaminocalcein (DCl-DA Cal) as a novel fluorescence probe for $\mathrm{NO}$, to confirm that improving the retention of fluorescence probes generally leads to enhancement of sensitivity [9]. The fluorescence quantum yield of DCl-DA

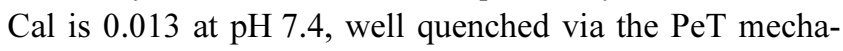
nism. When DCl-DA Cal reacts with NO in air, the triazole compound, DCl-DA Cal T, will be produced and will emit strong fluorescence (Fig. 4).

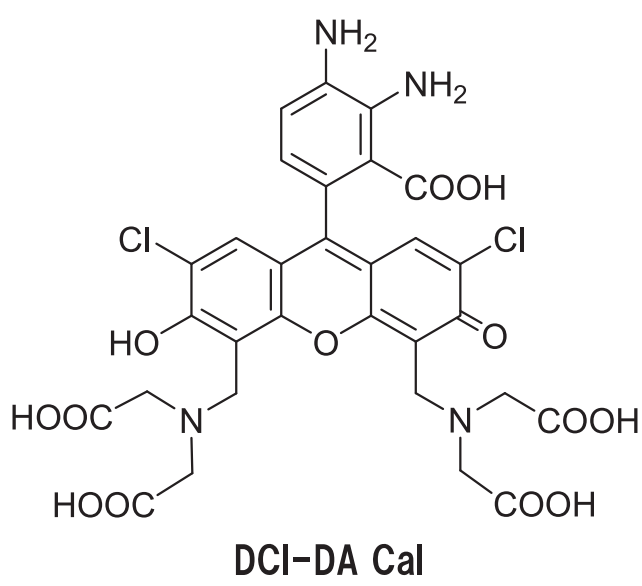

Fig. 4. Structure of DCl-DA Cal compound
The increase of fluorescence intensity of DCl-DA Cal is attributable to the formation of $\mathrm{DCl}-\mathrm{DA} \mathrm{Cal} \mathrm{T}$ in the reaction with NO. The fluorescence intensity of DCl-DA Cal T is high in the physiological range and its fluorescence quantum yield is 0.64 at $\mathrm{pH}$ 7.4. As a membrane-permeable fluorescence probe, we also prepared DCl-DA Cal-AM, in which the phenolic hydroxyl group and carboxyl group are protected as acetoxymethyl ester.

To examine how well DCl-DA Cal is retained in living cells, we compared DCl-DA Cal-AM with DAF-2 DA and DAF-4 DA. The experiment to examine the leakage of these triazole compounds, DCl-DA Cal T, DAF-2 T and DAF-4 T, was done. In contrast to rapid leakage of DAF-2 $\mathrm{T}$ and DAF-4 T, DCl-DA Cal-T was well retained in the living cells. The results obtained suggested that DCl-DA Cal and DCl-DA Cal T have excellent intracellular retention.

We applied DCl-DA Cal-AM to cultured bovine aortic endothelial cells. The results indicated that visualization of low levels of NO depends critically on the lack of leakage of the fluorescence probes and the fluorescence product from the cells.

In order to develop fluorescence probes that offer high sensitivity inside living cells, efficient retention of the probes and products within the cells is required. The IAG of calcein was very effective in improving intracellular retention. These fluorescence probes and their fluorescent products show good intracellular retention compared with conventional fluorescence probes, and are suitable for the visualization of low levels of physiological molecules and for long-term observation in living cells.

\section{BODIPY-based probe: DAMBO- $P^{H}$}

Boron dipyrromethene (BODIPY) is known to have a high quantum yield $\left(\Phi_{\mathrm{fl}}\right)$ of fluorescence in aqueous solution but has not been utilized much for biological applications, compared to fluorescein. We developed DAMBO-P ${ }^{\mathrm{H}}$, based on the BODIPY chromophore, as a highly sensitive fluorescence probe for NO [10] (Fig. 5). DAMBO-P ${ }^{\mathrm{H}}$ had a low $\Phi_{\mathrm{fl}}$ value of 0.002 , whereas its triazole derivative (DAMBO- $\mathrm{P}^{\mathrm{H}}$<smiles></smiles>

Fig. 5. Structure of DAMBO- $\mathrm{P}^{\mathrm{H}}$ compound 
$\mathrm{T})$, the product of the reaction of DAMBO- $\mathrm{P}^{\mathrm{H}}$ with $\mathrm{NO}$, fluoresced strongly $\left(\Phi_{\mathrm{fl}}=0.74\right)$. The change of the fluorescence intensity is controlled by an intramolecular PeT mechanism. The strategy for development of DAMBO- $\mathrm{P}^{\mathrm{H}}$ was as follows: (1) in order to design a highly sensitive probe of NO, o-phenylenediamine derivatives as NOreactive moieties was used as in the case of DAF-2, (2) in order to avoid $\mathrm{pH}$-dependency of the fluorescence intensity, the PeT process was controlled by modulating the spectroscopic and electrochemical properties of BODIPY chromophores according to the Rehm-Weller equation, (3) in order to avoid quenching of fluorescence by stacking of the probes and to obtain probes suitable for biological applications, hydrophilic functional groups were introduced. This strategy should be applicable for the rational design of other novel and potentially useful bioimaging fluorescence probes. Thus, we succeeded in developing a novel NO probe based on BODIPY structure.

\section{Diaminocyanines: DACs}

We have successfully developed several fluorescent probes (DANs, DAFs, DARs DCl-DA Cal and DAMBO- $\mathrm{P}^{\mathrm{H}}$ ) for $\mathrm{NO}$, and these probes have been widely used in biological applications. However, they have severe limitations with regard to ex vivo and in vivo applications to detect $\mathrm{NO}$ in isolated organs since their fluorescence lies in the visible region around $500-550 \mathrm{~nm}$, which cannot penetrate deeply into human tissues. While visible light is highly absorbed by biological substances such as hemoglobin, near-infrared (NIR) light at around 650-900 $\mathrm{nm}$ is less absorbed by such molecules and can penetrate more deeply into tissues. Moreover, it has the further advantage that autofluorescence is not observed upon NIR excitation. For these reasons, NIR fluorescence imaging is potentially very attractive for in vivo imaging. This section presents novel NIR fluorescent probes which are highly sensitive to NO and establish their utility by imaging NO in isolated intact rat kidneys (Fig. 6).

The probes are composed of two moieties: tricarbocyanine as the NIR fluorophore, which has a high extinction coefficient of about $1.5-2.0 \times 10^{5} \mathrm{M}^{-1} \mathrm{~cm}^{-1}$, and $o$-phenylenediamine as the NO-sensitive fluorescence

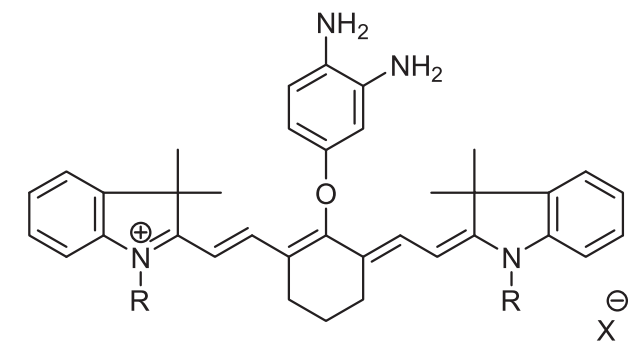

$$
\begin{aligned}
& \text { DAC-P : } \mathrm{R}=\mathrm{CH}_{2} \mathrm{CH}_{2} \mathrm{CH}_{3}, \mathrm{X}=\mathrm{I} \\
& \text { DAC-S : } \mathrm{R}=\mathrm{CH}_{2}\left(\mathrm{CH}_{2}\right)_{3} \mathrm{SO}_{3} \mathrm{Na}, \mathrm{CH}_{2}\left(\mathrm{CH}_{2}\right)_{3} \mathrm{SO}_{3}{ }^{-}
\end{aligned}
$$

Fig. 7. Structures of DAC compounds

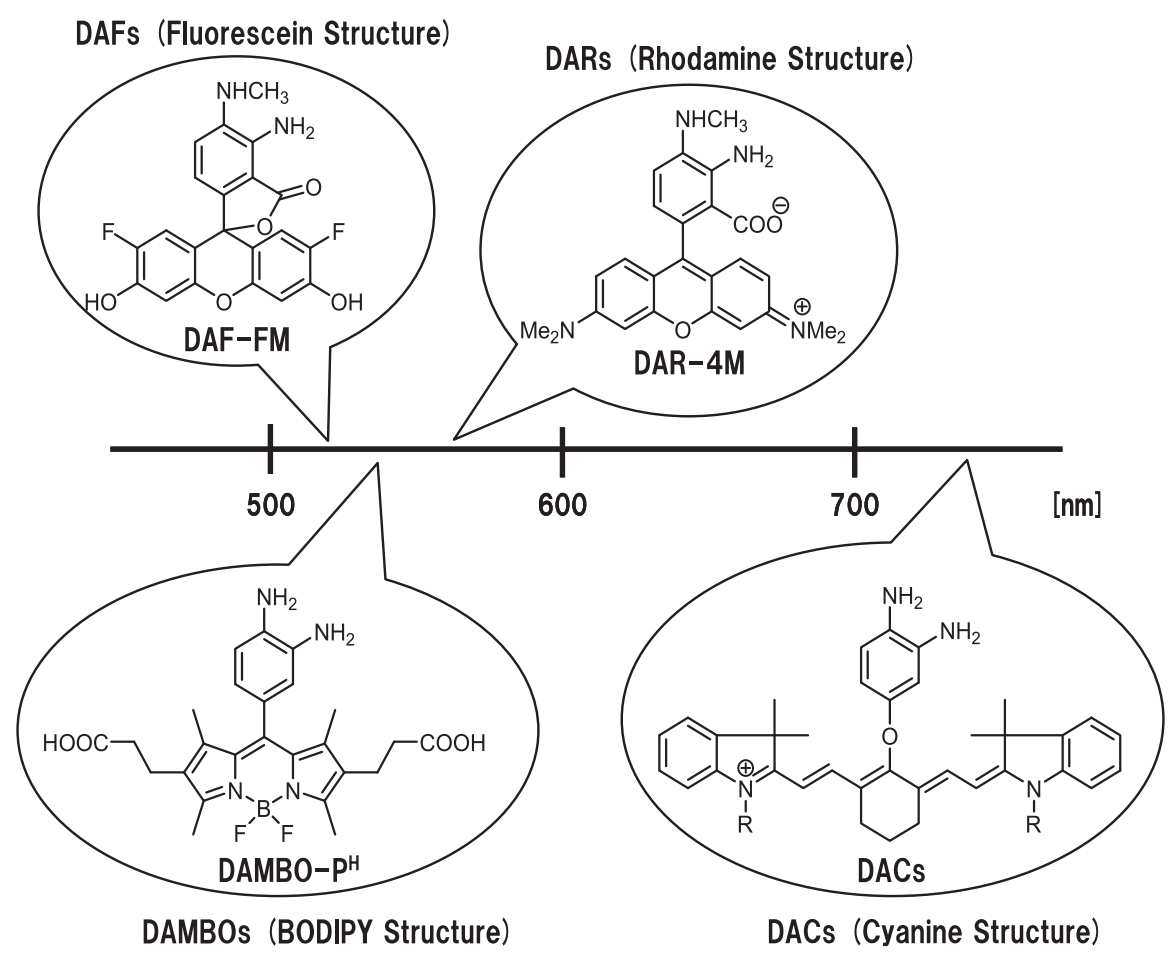

Fig. 6. Various NO probes with different emission wavelengths 
modulator. We named these new probes diaminocyanines (DACs), and the corresponding triazole compounds DAC-Ts [11]. They can be classified into two types; DAC-P with two propyl groups was designed to penetrate cellular membranes without any modification, and DAC-S with sulfonate groups was expected to be highly soluble in water. Consequently, the appropriate type can be selected for particular purposes (Fig. 7).

Their NIR fluorescence was increased in an NO concentration-dependent manner under physiological conditions, and their reaction efficiency with NO was at least 53 times higher than that of a widely used NO probe, DAF-2. They were confirmed to function in isolated intact rat kidneys. We applied DAC to isolated rat kidneys to examine whether it worked in ex vivo biological systems and whether we could observe the fluorescence change from outside the kidney without making sections. We selected DAC-P because it should be loaded more readily than DAC-S. Kidneys from male Wistar rats were isolated and perfused. As we expected, DAC-P was easily loaded into the kidneys simply by administering it into the right renal artery with the perfusate for several minutes and was hardly washed out throughout the observation. Then, an NO donor (NOC 13) was administered in the same manner. We observed a fluorescence increase during the administration of NOC 13. That is to say, DAC-P functions in ex vivo biological systems, and we could detect its fluorescence from outside the kidney. We noticed many circular patterns with a diameter of approximately $0.2 \mathrm{~mm}$ on the fluorescence images. We confirmed that these patterns corresponded to the glomeruli inside the kidney by observing the fluorescence images of renal sections. The observations are consistent with the structure of the glomeruli, which are covered with reticular blood vessels. DAC-P should be loaded into the endothelial cells during the perfusion. Thus, we succeeded in imaging $\mathrm{NO}$ in the isolated rat kidney using DAC-P.

\section{Probe for Peroxynitrite: NiSPY}

In the last 20 years, the study of aromatic nitration has been extended to biological events, because nitrated biomolecules have been implicated in various pathogenic processes, including inflammatory events, ischemia-reperfusion

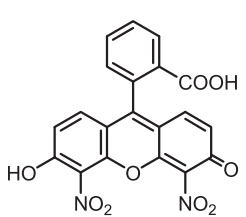

1

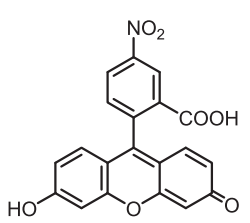

2

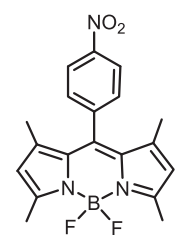

3
Fig. 8. Structures of fluorophores with nitro functional group and neurodegenerative disorders. $\mathrm{ONOO}^{-}$is considered to be a potent nitrating agent generated by the reaction of $\mathrm{O}_{2}{ }^{--}$ with NO in biological systems. Though it is tremendously significant to study the role of nitrative stress induced by $\mathrm{ONOO}^{-}$in biological systems, no fluorescent probe for monitoring $\mathrm{ONOO}^{-}$in biological samples has yet been developed.

In the design of novel fluorescent probes for $\mathrm{ONOO}^{-}$, a major obstacle must be overcome, i.e., the nitro group is believed to be a strong quencher of fluorescent dyes. Indeed, nitro-substituted fluorescein and BODIPY derivatives are almost non-fluorescent. The quenching mechanism of the nitro group is unclear, and some researchers have suggested that the nitro group has a unique action. However, we considered that the nitro group would have no unique effect, other than its electron-withdrawing effect, on an adjacent fluorophore, and that the fluorescence quantum efficiency $\left(\Phi_{\mathrm{fl}}\right)$ can be precisely predicted.

To test our hypothesis, we firstly focused on the structure of nitro-substituted dyes (Fig. 8). From the viewpoint of fluorescence, the structures of fluorescein and BODIPY can be divided into two parts, the xanthene or BODIPY moiety as a fluorophore and the benzene moiety as a fluorescence switch which modulates the $\Phi_{\mathrm{fl}}$ value of fluorophore, since they are orthogonal to each other. In the case of 1 in Fig. 8, the $\pi$ electron system and transition process of the fluorophore are significantly perturbed by a directly conjugated nitro group, and as a consequence of these perturbations, 1 becomes almost non-fluorescent. On the other hand, in the cases of 2 and 3 , the nitro group is not directly conjugated to the fluorophore, but is located at a proximal position. Therefore, unlike 1 , there should be no $\pi$ electron conjugation between the fluorophore and the nitro group, since the benzene moiety and the fluorophore are uncoupled. In this case, how does the adjacent nitro group significantly affect the fluorescence properties of the fluorophore?

To address this question, we next focused on the LUMO energy level of nitro-substituted aromatic rings. In general, nitro groups greatly lower the LUMO energy level of aromatic compounds due to their strong electron-withdrawing effect. We have reported [12] that an electron-deficient benzene moiety can quench the fluorescence of a fluorophore via an intramolecular PeT process from the excited fluorophore to the electron-deficient benzene moiety (donorexcited PeT; d-PeT). According to this principle, in the cases of 2 and 3, the benzene moiety should act as an electron acceptor for the excited fluorophore due to the strong electron-withdrawing effect of the nitro group, and so we hypothesized that the most plausible mechanism for the quenching of the fluorescence of 2 and 3 is the d-PeT process.

Based on the above concept, it should be possible to design a highly fluorescent compound, even if it has a nitro

Vol. 45, No. 2, 2009 
<smiles></smiles>

NiSPY-1: $\mathbf{R}^{1}=\mathrm{CH}_{3}, \mathbf{R}^{2}=\mathrm{CH}_{3}$

NiSPY-2 : $\mathbf{R}^{1}=\mathrm{H}, \mathrm{R}^{2}=\mathrm{CH}_{3}$ NiSPY $-3: \mathrm{R}^{1}=\mathrm{H}, \mathrm{R}^{2}=\mathrm{CH}_{2} \mathrm{COOH}$

Fig. 9. Structures of NiSPY compounds

group proximal to the fluorophore, by appropriately controlling the relative free energy change of the PeT process $(\Delta \mathrm{GeT})$. To test this hypothesis, we designed and synthesized several nitroBODIPY derivatives. As the results, we had succeeded in designing an extremely rare example of a highly fluorescent compound bearing a nitro group by utilizing a PeT mechanism-based approach. Now, we can understand that the nitro group simply acts as an electronwithdrawing group. This is the key to the design of novel fluorescence probes for nitrative stress. With this information in hand, we designed our fluorescence probes for nitrative stress (NiSPYs) [13].

To examine whether NiSPY-1 (Fig. 9) can monitor the nitration reaction or not, we examined the reaction between NiSPY-1 and $\mathrm{NO}_{2} \mathrm{BF}_{4}$, which serves as a nitronium source. Nitration reaction of the benzene moiety of NiSPY-1 proceeded rapidly in $\mathrm{CH}_{3} \mathrm{CN}$, and the fluorescence of NiSPY-1 was dramatically enhanced. Next, we tried to detect $\mathrm{ONOO}^{-}$in aqueous media with NiSPY- $1 . \mathrm{ONOO}^{-}$is a potent nitrating agent which is generated by the reaction of $\mathrm{O}_{2}{ }^{--}$ with NO in biological systems. Unfortunately, NiSPY-1 could not detect $\mathrm{ONOO}^{-}$, due to its low reactivity and low water solubility. We therefore made some structural modifications of NiSPY-1 to generate higher reactivity and water solubility, while retaining the desired fluorescence off/on properties before and after the nitration. To provide higher reactivity, we employed a phenol derivative as the benzene moiety, since it is known to be more reactive with $\mathrm{ONOO}^{-}$ compared with anisole derivatives. Water solubility was improved by incorporating a carboxyl group in the benzene moiety, and thus we obtained the novel fluorescence probes, NiSPY-2 and NiSPY-3 (Fig. 9). As expected, the fluorescence of NiSPY-2 and NiSPY-3 was dramatically enhanced upon addition of $\mathrm{ONOO}^{-}$. Moreover, NiSPY-3 showed little fluorescence augmentation upon addition of other ROS, such as ${ }^{\circ} \mathrm{OH}, \mathrm{HOCl},{ }^{1} \mathrm{O}_{2}, \mathrm{NO}, \mathrm{O}_{2} \cdot{ }^{--}$and $\mathrm{H}_{2} \mathrm{O}_{2}$, whereas strong fluorescence enhancement was observed upon reaction with $\mathrm{ONOO}^{-}$. NiSPY was applicable for live cell fluorescence imaging of $\mathrm{ONOO}^{-}$in SIN-1 (a compound that releases $\mathrm{ONOO}^{-}$) loaded HeLa cell with no apparent cell toxicity.

\section{Probes for Highly Reactive Oxygen Species (hROS)}

\section{$H P F$ and $A P F$}

There are many species of ROS, as mentioned in the Introduction Section, but they tend to be considered collectively as "oxidative stress" when their effects in living cells are discussed. However, we believe that each species of ROS is likely to have a specific role in living cells. For example, $\mathrm{H}_{2} \mathrm{O}_{2}$ is an endothelium-derived hyperpolarizing factor in human and mice, p38 mitogen-activated protein kinase mediates caspase- 3 activation during apoptosis induced by ${ }^{1} \mathrm{O}_{2}$ but not by $\mathrm{H}_{2} \mathrm{O}_{2}$, and ${ }^{\circ} \mathrm{OH}$ plays an important role as a second messenger in $\mathrm{T}$ cell activation. In addition, each species of ROS has a characteristic chemical reactivity; for example, ${ }^{1} \mathrm{O}_{2}$ reacts with anthracenes to yield endoperoxides in the Diels-Alder mode, whereas ${ }^{\circ} \mathrm{OH}$ can react directly with aromatic rings to yield hydroxylated products, and NO reacts with guanine to yield the deaminated compound. However, due to the lack of selectivity of detection methods among ROS, the roles of an individual species of ROS in living cells remain uncertain.

If novel fluorescence probes that overcome the above problems are available, they would contribute greatly to the elucidation of the roles of individual ROS in living cells, because we would be able to "see" the generation of specific ROS with high resolution in time and space.

It is known that ${ }^{\circ} \mathrm{OH}$ participates in various biological processes. For example, HeLa, MW451, and HL-60 cells are induced to undergo apoptosis by ${ }^{\circ} \mathrm{OH}$. $\cdot \mathrm{OH}$ can damage DNA bases and mediates redox alteration of cell-membrane $\mathrm{Ca}^{2+}$ channels. However, because of the lack of effective direct detection methods for ${ }^{\circ} \mathrm{OH}$, its participation in these events has been established only indirectly by using inhibitors such as dithioethanol, glutathione, and desferrioxamine. Therefore, we wished to develop novel fluorescence probes for highly ROS (hROS). Here, we use the term hROS to indicate ROS with strong oxidizing power sufficient to directly hydroxylate aromatic rings, such as ${ }^{\circ} \mathrm{OH}$ or reactive intermediates of peroxidase.

Several fluorescence probes to detect ROS, such as 2',7'dichlorodihydrofluorescein (DCFH) and dihydrorhodamine 123, have also been developed. However, as Hempel and co-workers [14] pointed out, DCFH and dihydrorhodamine 123 can react with various ROS and oxidizing species including $\mathrm{O}_{2}^{--}, \mathrm{H}_{2} \mathrm{O}_{2}, \mathrm{NO}$, ferrous ion, and others, and in addition, DCFH is easily autoxidized, resulting in a spontaneous increase in fluorescence upon exposure to light. Therefore, it is not appropriate to think of these probes as 


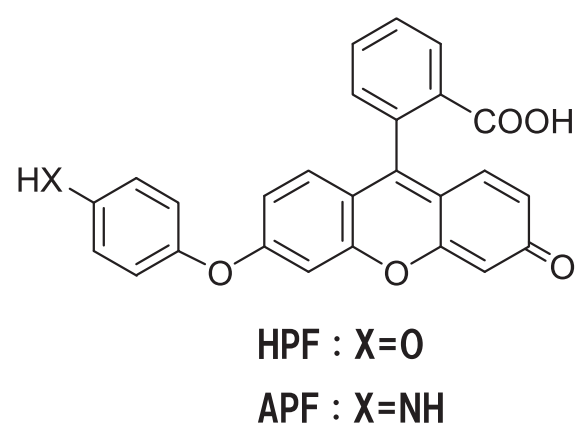

Fig. 10. Structures of HPF and APF

detecting a specific oxidizing species in cells, such as $\mathrm{H}_{2} \mathrm{O}_{2}$ or NO, but rather they should be considered as detecting a broad range of oxidizing reactions that may be increased during intracellular oxidative stress.

We will show the chemical reaction as the basis of probe design. We reported previously that aryloxyphenols are Odearylated in the ipso-substitution manner by hROS such as $\cdot \mathrm{OH}$, reactive intermediates of peroxidase and cytochrome $\mathrm{P} 450$, but not by other $\operatorname{ROS}\left(\mathrm{O}_{2}{ }^{--}, \mathrm{H}_{2} \mathrm{O}_{2},{ }^{1} \mathrm{O}_{2}\right.$, and so on). Based on the reaction, we designed and synthesized HPF and APF as novel fluorescence probes to detect selectively hROS such as ${ }^{\circ} \mathrm{OH}$ and reactive intermediates of peroxidase [15] (Fig. 10).

We expected that almost non-fluorescent HPF and APF would be O-dearylated upon reaction with hROS to yield strongly fluorescent fluorescein. As expected, the results indicate that HPF and APF themselves scarcely fluoresce, while APF selectively and dose-dependently afford a strongly fluorescent compound, fluorescein, upon reaction with hROS, $\mathrm{ONOO}^{-}$or $\mathrm{HOCl}$, but not other ROS. HPF similarly afforded fluorescein upon reaction with hROS or $\mathrm{ONOO}^{-}$, not with $\mathrm{HOCl}$ or other ROS. Therefore, not only can hROS and $\mathrm{ONOO}^{-}$be differentiated from $\mathrm{H}_{2} \mathrm{O}_{2}, \mathrm{NO}$, and $\mathrm{O}_{2}{ }^{--}$by using HPF or APF alone, but $\mathrm{HOCl}$ can also be specifically detected by using HPF and APF together. We also found that HPF and APF are resistant to light-induced autoxidation, unlike DCFH.

\section{$A P C$}

HPF and APF probes are the useful probe for detection of hROS, however they may be unsuitable for continuous and prolonged observation, because they leak quite readily from living cells. In short, the concentration of fluorescein-based fluorescence probes cannot be maintained at a sufficiently high level in living cells, and this impairs the achievable sensitivity. Actually, it has been found that HPF and APF probes cannot detect low level of hROS production in living cells due to the high leakage.

To overcome this problem, we examined the intracellular retention of several well-known fluorescent molecules,

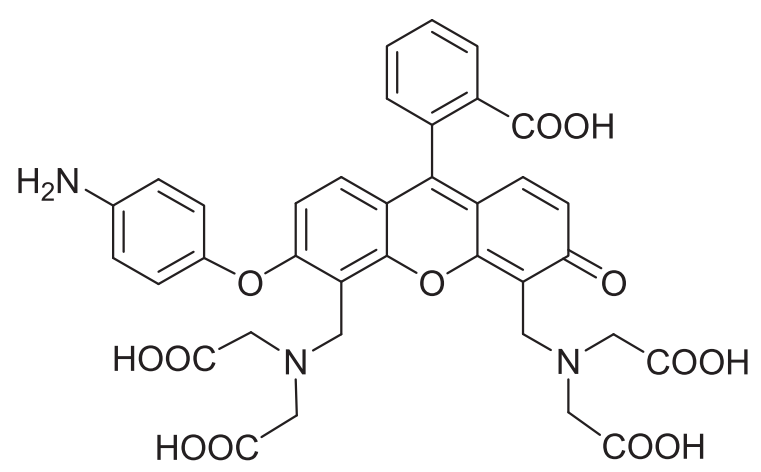

APC

Fig. 11. Structure of APC

calcein, BCECF, carboxyfluorescein and fluorescein, as well as some novel derivatives, based on the strategy shown in the section of DCl-DA Cal. From the results obtained, we considered that calcein, which contains two IAGs, would be an excellent scaffold of fluorescence probes. The calcein scaffold should allow highly sensitive detection and prolonged observation in living cells. First, we investigated the chemical characteristics of calcein. Its electrochemical features were examined with cyclic voltammetry in order to see whether or not the PeT mechanism can be applied to calcein. The results suggested that the fluorescence of calcein can also be controlled by the PeT mechanism. We also confirmed that the IAGs of calcein serve to make the fluorescence $\mathrm{pH}$-insensitive in the physiological $\mathrm{pH}$ range, while retaining strong fluorescence intensity.

Next, we utilized these findings to develop superior fluorescence probes. As shown in HPF and APF Section, aryloxyphenols quench the fluorescence of fluorescein via the PeT mechanism, and are O-dearylated in the ipsosubstitution manner by hROS, such as ${ }^{\circ} \mathrm{OH}, \mathrm{ONOO}^{-}$and $\mathrm{HOCl}$.

So, we designed and synthesized a novel fluorescence probe, aminophenoxycalcein (APC) [9], which was expected to have excellent intracellular retention and not to show $\mathrm{pH}$ sensitivity of the fluorescence intensity under physiological conditions (Fig. 11).

We evaluated the selectivity of APC for various ROS. APC showed a fluorescence increase only upon reaction with ${ }^{\circ} \mathrm{OH}, \mathrm{ONOO}^{-}$, and $\mathrm{HOCl}$. Furthermore, the fluorescence intensity reached the maximum immediately, and also showed a dose-dependent increase upon addition of hROS. On the other hand, in the presence of other ROS, there was no change in the fluorescence intensity. These results demonstrate that APC has high selectivity for hROS. The $\mathrm{HRP} / \mathrm{H}_{2} \mathrm{O}_{2}$ system is well known to generate hROS, and APC could also detect hROS in this system.

Because APC lacks membrane permeability, we synthesized the acetoxymethyl ester derivative, APC-AM, which 


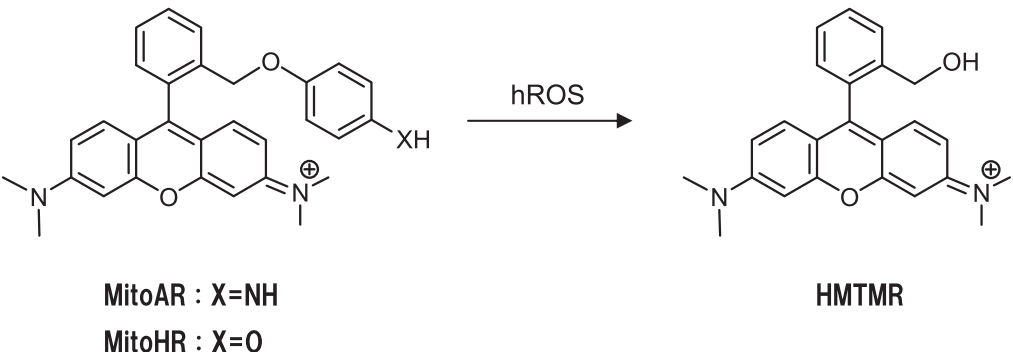

Fig. 12. Structures of MitoAR and MitoHR and reaction with hROS

was expected to be membrane-permeable, and applied it to HL-60 cells, which are known to produce $\mathrm{HOCl}$ upon stimulation. In the case of APF, the fluorescence intensity initially increased upon stimulation, and decreased after 10 min. With APC-AM, on the other hand, the fluorescence intensity continued to increase for over $30 \mathrm{~min}$, and became much more intense than that of APF. This was considered to be due to effective intracellular retention of APC and calcein.

The characteristics required for a superior fluorescence probe include not only a large fluorescence increase, but also stability of fluorescence intensity under physiological conditions. We applied APC-AM to image hROS inside HL-60 cells by means of fluorescence microscopy. APCAM could detect even a small amount of hROS which was difficult to distinguish from noise with APF.

In conclusion, in order to develop more practically useful fluorescence probes, we focused on the leakage of the probes from living cells. We found that the IAGs of calcein are very useful both to improve intracellular retention and to reduce the $\mathrm{pH}$ sensitivity of fluorescence intensity under physiological conditions.

\section{MitoAR}

Mitochondria are believed to be the major source of intracellular ROS. $\mathrm{O}_{2}^{--}$is first generated in the respiratory chain and can be converted to $\mathrm{H}_{2} \mathrm{O}_{2}$ in the intermembrane space. Even though $\mathrm{O}_{2}{ }^{--}$and $\mathrm{H}_{2} \mathrm{O}_{2}$ are not strong oxidants, they are precursors of hROS such as ${ }^{\circ} \mathrm{OH}, \mathrm{ONOO}^{-}, \mathrm{HOCl}$. In the presence of transitional metal complexes, for example, iron or copper complexes, which are rich in the mitochondrial inner membrane, $\mathrm{H}_{2} \mathrm{O}_{2}$ can be converted to the highly reactive ${ }^{\circ} \mathrm{OH}$ via Fenton reaction. $\mathrm{O}_{2}{ }^{--}$can react with $\mathrm{NO}$, formed by mitochondrial nitric oxide synthase, to generate $\mathrm{ONOO}^{-}$. hROS are strong oxidants that can directly oxidize DNAduplex, proteins and lipids. Despite their importance, however, the precise roles of mitochondrial hROS are still elusive.

One reason for this is that no method is yet available for direct detection of hROS selectively in mitochondria. Dihydrorhodamine has been widely used in conjunction with fluorescence microscopy to detect ROS. In general, a positive charge facilitates movement across phospholipid bilayers and accumulation into the mitochondrial matrix in response to the negative membrane potential, but dihydrorhodamine is not cationic before oxidation, and so would not be localized preferentially in mitochondria. It is therefore unsuitable for selective detection of ROS generation in mitochondria. Furthermore, dihydrorhodamine is extensively autoxidized, resulting in a marked increase of the fluorescence intensity. Therefore, there is a need to develop novel mitochondria-selective probes for hROS.

For this purpose, we chose rhodamine dye as a fluorophore, since it is already used as a probe for mitochondria. Rhodamine dye has a positive charge, as well as many other favorable characteristics for biological applications, including high water solubility, high fluorescence intensity, long excitation and emission wavelengths $(>500 \mathrm{~nm}), \mathrm{pH}$ independent fluorescence, and tolerance to photobleaching. In short, it has excellent photochemical properties and is suitable for fluorescence microscopy studies.

As shown in HPF and APF Section, 4-aminophenyl aryl ether and 4-hydroxylphenyl aryl ether react with hROS selectively over other ROS. 4-Amino- or 4-hydroxylphenyl ether moieties are more electron-rich than the xanthene moiety and we considered that they could be used to regulate the fluorescence of rhodamine through PeT process. To facilitate the PeT process, we designed molecules in which the electron donor (4-amino- or 4-hydroxyphenyl ether) was placed at the 2-position of the phenyl moiety of rhodamine, thereby being fixed in close proximity to the xanthene structure. Consequently the fluorescence of the dye should be efficiently quenched. Reaction with hROS should cause cleavage of the ether moiety, affording hydroxymethyltetramethylrhodamine (HMTMR), which is highly fluorescent in an aqueous environment. Thus, we synthesized the two novel fluorescent probes MitoAR and MitoHR, which were expected to be selectively localized in mitochondria, and to react selectively with hROS [16] (Fig. 12).

When MitoAR and MitoHR reacted with hROS $\left({ }^{\circ} \mathrm{OH}\right.$, $\mathrm{ONOO}^{-}, \mathrm{HOCl}$ ), a large and immediate increase of fluorescence intensity was observed. In the case of MitoAR, the 
fluorescence intensities reached the maximum within a few seconds, whereas the reactions with other $\operatorname{ROS}\left(\mathrm{O}_{2}{ }^{-}, \mathrm{NO}\right.$, $\mathrm{H}_{2} \mathrm{O}_{2}$ ) led to almost no fluorescence increase even after 30 min. Thus, MitoAR and MitoHR appear to be a highly selective for the detection of hROS. In addition, we examined the fluorescence response of MitoAR with enzymatic systems including an $\mathrm{HRP} / \mathrm{H}_{2} \mathrm{O}_{2}$ system, to cause intense increase of the fluorescence intensity. In an $\mathrm{O}_{2}{ }^{-{ }^{-}}$generating $\mathrm{X} / \mathrm{XO}$ system, addition of xanthine to MitoAR and xanthine oxidase produces a much smaller fluorescence increase about 1.1fold.

We next used confocal microscopy to assess the localization of MitoAR and its ability to sense hROS in HeLa cells. We selected MitoAR for this purpose because it has higher reactivity for hROS and better photochemical properties than MitoHR. First, we co-stained HeLa cells with MitoAR and MitoTracker Green FM, a mitochondrial stain. MitoAR is almost, but not completely, nonfluorescent and could be detected with the microscope. The fluorescence images of the two dyes were well merged, which indicates that MitoAR is localized selectively in mitochondria. Next, $\mathrm{NaOCl}$ was added to MitoAR-loaded HeLa cells to examine whether MitoAR could detect hROS in living cells. The addition of $\mathrm{NaOCl}$ resulted in a large increase of fluorescence. There appears to be some diffusion of dye out of the mitochondria after reaction with $\mathrm{NaOCl}$. This could be due to a loss of mitochondrial membrane potential after $\mathrm{NaOCl}$ treatment, allowing a small part of the resulting fluorescent product to leak out. These results showed that MitoAR has the ability to detect hROS in mitochondria.

With these results in hand, we examined hROS generation inside the cells. When we stimulated MitoAR-loaded HeLa cells with $\mathrm{H}_{2} \mathrm{O}_{2}$, which does not react with MitoAR at all, no fluorescent increase was observed even at the concentration of $1 \mathrm{mM}$. However, in the case of MitoAR-loaded HL-60 cells stimulated with $100 \mu \mathrm{M} \mathrm{H}_{2} \mathrm{O}_{2}$, the fluorescence intensity increased gradually and strong fluorescence was seen after $10 \mathrm{~min}$. HL-60 cells, but not HeLa cells, contain myeloperoxidase in azurophilic granules, and $\mathrm{H}_{2} \mathrm{O}_{2}$ activates myeloperoxidase to produce $\mathrm{HOCl}$, which is generated outside the mitochondria. This is presumably the reason why only HL-60 cells showed a large fluorescence increase. Thus, different cell types appear to generate different levels of hROS in response to $\mathrm{H}_{2} \mathrm{O}_{2}$-induced stress, and MitoAR can detect this with high sensitivity.

\section{Probes for Singlet Oxygen: DPAX and DMAX}

${ }^{1} \mathrm{O}_{2}$, an excited state of molecular oxygen, is one of the active species for oxidation in biological systems, together with $\mathrm{O}_{2}{ }^{-}$, ${ }^{\circ} \mathrm{OH}, \mathrm{H}_{2} \mathrm{O}_{2}$, etc. Many researchers have investigated the reactivity of ${ }^{1} \mathrm{O}_{2}$ toward organic substrates, as well as its possible role in biological systems. ${ }^{1} \mathrm{O}_{2}$ is considered to be an important toxic species in vivo since it can oxidize DNA, proteins and lipids, and its reactivity toward DNA bases has been especially well characterized. In addition, ${ }^{1} \mathrm{O}_{2}$ is reported to play a role as an activator of gene expression.

Although many ${ }^{1} \mathrm{O}_{2}$ traps have been reported, it is still difficult to detect ${ }^{1} \mathrm{O}_{2}$ generated in biological systems because of high reactivity and its short lifetime ( $2 \mu$ s in water). The most widely used ${ }^{1} \mathrm{O}_{2}$ trap is 9,10-diphenylanthracene (DPA), which reacts rapidly with ${ }^{1} \mathrm{O}_{2}$ specifically to form a thermostable endoperoxide. The decrease in absorbance at $355 \mathrm{~nm}$ is used as a measure of the formation of the endoperoxide. Many water-soluble DPA derivatives have been developed, but the quenching of ${ }^{1} \mathrm{O}_{2}$ by water means that they are difficult to apply to biological systems. DPA derivatives are not very sensitive as probes because the detection is based on the measurement of absorbance. Some results are still controversial, mainly because of the lack of a reliable detection method. Hence, we designed and synthesized novel fluorescent probes for ${ }^{1} \mathrm{O}_{2}$ in order to improve the sensitivity. In general, fluorescence measurement is more sensitive, and so is easier to use in imaging studies.

We designed DPAX-1 as a suitable fluorescent probe [17], which is fused with DPA and fluorescein. When DPAX reacts with ${ }^{1} \mathrm{O}_{2}$ to yield DPAX-endoperoxide (DPAX-EP), the conjugation between the DPA structure and xanthene ring is greatly altered, so we expected a change in fluorescence properties based on the PeT mechanism. In order to stabilize the fluorescence intensity at physiological $\mathrm{pH}$ we incorporated electron-withdrawing groups, $\mathrm{Cl}$ (DPAX-2) and F (DPAX-3), at the 2- and 7-positions of the xanthene chromophore. Absorbance maxima, molar absorption coefficients, and emission maxima were not altered much between DPAXs and DPAX-EPs. However, the quantum efficiencies of fluorescence $\left(\Phi_{\mathrm{fl}}\right)$ were greatly changed: DPAXs themselves are almost non-fluorescent and DPAXEPs are highly fluorescent. The maximum absorbance and emission of the chlorinated compound DPAX-2-EP were shifted to longer wavelengths than the other compounds. Tthe $\mathrm{pH}$ profiles of fluorescence intensity for the DPAX-EPs showed that DPAX-2 and DPAX-3 are useful as probes under neutral conditions, compared to DPAX-1. Then, the fluorescence intensity of DPAX-2 increased with the generation of ${ }^{1} \mathrm{O}_{2}$ using 3-(4-methyl-1-naphthyl) propionic acid endoperoxide (EP-1) or $\mathrm{Mo}^{6+}-\mathrm{H}_{2} \mathrm{O}_{2}$ system as a ${ }^{1} \mathrm{O}_{2}$ source in a dose-dependent manner. The fluorescence intensity of DPAX-2 did not change upon reaction with $\mathrm{H}_{2} \mathrm{O}_{2}, \mathrm{O}_{2} \cdot-$ and NO, which confirms the specificity of DPAX-2 for ${ }^{1} \mathrm{O}_{2}$ (Fig. 13).

DPAXs are useful for detection of ${ }^{1} \mathrm{O}_{2}$, however they have some limitations in application to biological systems, especially with regard to their sensitivity. We designed a novel fluorescence probe with much higher sensitivity for 


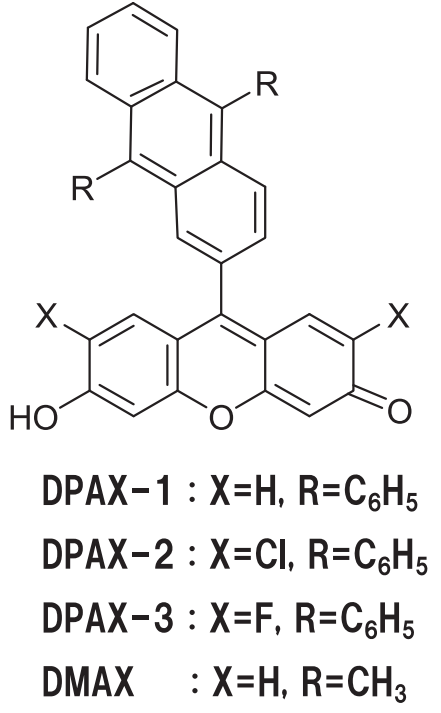

Fig. 13. Structure of DPAXs and DMAX compounds

${ }^{1} \mathrm{O}_{2}$, with a faster rate of formation of endoperoxide upon reaction with ${ }^{1} \mathrm{O}_{2}$ (DMAX) [18]. This novel fluorescent probe has a 9,10-dimethylanthracene moiety as an extremely fast chemical trap of ${ }^{1} \mathrm{O}_{2}$. Similar to in the case of DPAXs, DMAX reacted rapidly with ${ }^{1} \mathrm{O}_{2}$ to produce DMAX endoperoxide (DMAX-EP) with strongly fluorescent intensity, but its sensitivity was 53 -fold higher than that of DPAXs. DMAX should be the best fluorescence reagent for ${ }^{1} \mathrm{O}_{2}$ detection currently available.

\section{Probe for Hypochlorous Acid: HySOx}

Neutrophils contain azurophilic granules, in which MPO exists abundantly, and MPO catalyzes the formation of hypochlorous acid ( $\mathrm{HOCl}$ ) from $\mathrm{H}_{2} \mathrm{O}_{2}$ and $\mathrm{Cl}^{-}$in vitro. $\mathrm{HOCl}$ has a strong microbicidal activity, and plays a key role in the killing of bacteria by neutrophils. In the presence of $\mathrm{Cl}^{-}$, $\mathrm{HOCl}$ is predominantly produced via the reactive intermediate, compound I. However, any specific detection methods for $\mathrm{HOCl}$ using fluorescent probes have not been reported until now. We developed HySOx as a specific fluorescent probe for $\mathrm{HOCl}$.

The tetramethylrhodamine (TMR) fluorophore is a useful fluorescent molecule, being applicable to biological investigations utilizing fluorescence microscopy, owing to its excellent photochemical properties in aqueous media, including high fluorescence intensity, longer excitation and emission wavelengths $(550-600 \mathrm{~nm})$, high molar extinction coefficient, independence of $\mathrm{pH}$ and tolerance to photobleaching, and its wide use as a fluorescent tag. However, it has not been widely employed in fluorescence probes, which are switched from a non-fluorescent to a fluorescent form upon interaction with analytes, in contrast to other common fluorophores such as fluorescein. One of the reasons for this is probably the lack of a good strategy to regulate the fluorescence of the TMR fluorophore. Therefore, a novel and widely applicable strategy to regulate the fluorescence of the TMR fluorophore would potentially be applicable to the design of probes for a wide range of analytes.

We previously reported [19] that the derivatization of fluorescein does not disrupt the photochemical properties when various functional groups are substituted for the carboxyl group at the 2 position. We thought that a similar molecular design strategy might be applicable to the TMR fluorophore, so we synthesized various 2'-substituted TMR derivatives, including a new TMR derivative, HMTMR (Fig. 12). To our surprise, the fluorescence of HMTMR showed a very interesting environmental dependence. HMTMR has a large absorbance and fluorescence in protic solvents but little in aprotic or basic solvents. This was found to be because of intramolecular spirocyclization at C9, deconjugating the TMR fluorophore. We obtained direct evidence of the spirocyclic form of HMTMR by X-ray crystallography. Rhodamines in a spirocyclic form have neither absorbance nor fluorescence in the visible region. So, regulation of the equilibrium between the spirocyclic form and the open circular form provides a strategy for controlling the fluorescence. Here, we have designed and developed a new fluorescence probe that works by regulating novel spirocyclization in physiological media.

The initial requirements for a practical fluorescence probe based on control of spirocyclization are that the probe molecule itself should be in almost $100 \%$ spirocyclic and non-fluorescent form, while the product of the interaction between probe molecule and analytes should be in an almost $100 \%$ open circular and fluorescent form. On the basis of this strategy, we designed and synthesized a thiol analogue of HMTMR to improve the stability of the spirocyclic structure in aqueous media at physiological $\mathrm{pH}$ [20].

The thiol analogue, HySOx (Fig. 14), was almost completely colorless and non-fluorescent in aqueous solutions over a wide range of $\mathrm{pH}$ values. HySOx can be isolated as a colorless solid from various organic solvents. HySOx favors a spirocyclic form more strongly than HMTMR because HySOx has a thiol group, which is more nucleophilic than the hydroxyl group of HMTMR. Thus, by regulating the nucleophilicity of the function at the 2 ' position, we obtained HMTMR, which has an open/closed equilibrium and is environmentally dependent, and HySOx, which favors a spirocyclic form independently of the environment and is completely non-fluorescent.

The thiol group works not only as a cyclization enhancer but also as a center of redox reaction. So, we tested the utility of HySOx as a fluorescence probe for ROS. Various important ROS generated in organisms (i.e., $\mathrm{HOCl},{ }^{\circ} \mathrm{OH}, \mathrm{ONOO}^{-}$, $\mathrm{NO}, \mathrm{O}_{2}{ }^{-},{ }^{1} \mathrm{O}_{2}$ and $\mathrm{H}_{2} \mathrm{O}_{2}$ ) were added to a solution of HySOx 

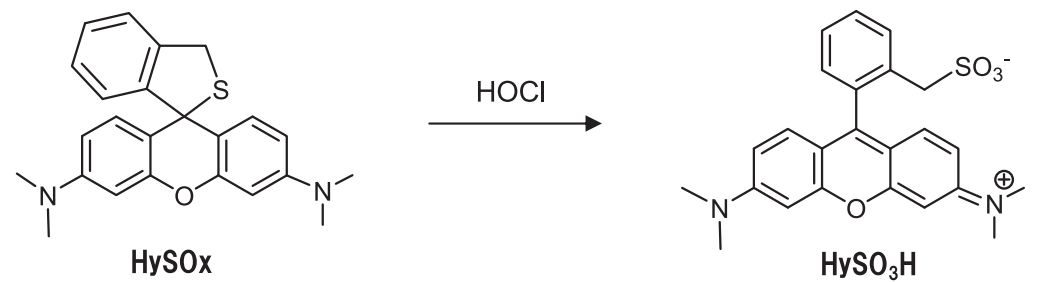

Fig. 14. Structure of $\mathrm{HySOx}$ and reaction with $\mathrm{HOCl}$ to produce $\mathrm{HySO}_{3} \mathrm{H}$

in $0.10 \mathrm{M}$ sodium phosphate buffer at $\mathrm{pH}$ 7.4. The fluorescence intensity increased quickly and quantitatively upon sodium hypochlotrite $(\mathrm{NaOCl})$ addition. Many oxidizing reagents are known to oxidize dihydrothiophene substrates in organic synthesis conditions. HySOx is able to detect $\mathrm{HOCl}$ specifically among various ROS in aqueous solution. There are other fluorescence probes that can detect $\mathrm{HOCl}$, but these probes detect other ROS, too. We also separated the main fluorescent product of the reaction between HySOx and $\mathrm{HOCl}$ and identified it as the sulfonate derivative, $\mathrm{HySO}_{3} \mathrm{H}$ (Fig. 14).

We next applied HySOx to visualize phagocytosis by porcine neutrophils, with opsonized zymosan derived from Saccharomyces cerevisiae as a target. HySOx detected $\mathrm{HOCl}$ generated inside the phagosomes just after the completion of phagocytosis. It is reported that the inside of the phagosome is acidic. We also confirmed that HySOx is able to detect $\mathrm{HOCl}$ specifically at $\mathrm{pH}$ 6.0. In the imaging of phagocytosis, there was little fluorescence increase due to autoxidation under laser excitation before phagocytosis began. Further, there was no fluorescence decrease due to photobleaching after membrane fusion following phagocytosis. HySOx has very superior properties as a probe, being tolerant to autoxidation due to its high specificity for $\mathrm{HOCl}$ and producing a fluorescent product, $\mathrm{HySO}_{3} \mathrm{H}$, which is photostable and $\mathrm{pH}$ independent owing to the TMR fluorophore. This probe, HySOx, can work in aqueous solution at $\mathrm{pH} 7.4$ and was confirmed to be able to detect $\mathrm{HOCl}$ being generated inside phagosomes in real time. Regulation of the spirocyclization of rhodamines provides a new approach to the rational development of novel fluorescence probes.

\section{Conclusions}

Noninvasive visualization and investigation of interactions among small biomolecules, proteins, DNA, lipids and sugar in living cells are an important goal for biologists, and fluorescence probes are powerful tools for this purpose. Because many target molecules are present in only trace amounts, high sensitivity is very important, and it is common to improve the sensitivity of fluorescence probes by focusing on high reaction velocity. So far, we have

\begin{tabular}{|c|c|c|c|c|c|c|}
\hline Probes RNS & $\cdot \mathrm{OH}$ & HOONO & $\mathrm{HOCl}$ & NO & $1 \mathrm{O}_{2}$ & Autooxidation \\
\hline HPF & 0 & 0 & & & & \\
\hline APF, APC, MitoAR & 0 & 0 & 0 & & & \\
\hline NiSPY & & 0 & & & & \\
\hline HySOx & & & 0 & & & \\
\hline $\begin{array}{l}\text { DAFs, DARs, } \\
\text { DCl-DA Cal } \\
\text { DAMBO-PH, DACs }\end{array}$ & & & & 0 & & \\
\hline DPAX, DMAX & & & & & 0 & \\
\hline DCFH & 0 & 0 & 0 & 0 & 0 & 0 \\
\hline
\end{tabular}

Fig. 15. Bioimaging probes for ROS and RNS

designed and synthesized various highly sensitive fluorescence probes based on PeT, spirocyclization, ICT and FRET. Nevertheless, they were sometimes insufficiently sensitive to detect biomolecules in living cells, despite high chemical sensitivity in cuvette. In this review, we introduced how to design bioimaging probes, which can detect various ROS and RNS in living systems. These novel fluorescence probes with high sensitivity should be useful as tools to study the role of ROS and RNS in biological samples and in pathological processes, because the probes enable us to confirm the generation of these functions, distinguishing it from other ROS and RNS (Fig. 15).

Our strategy should be applicable to develop fluorophorederived functional indicators for a wide range of applications. As with other imaging technologies, research on novel fluorescent probes should continue to be rapid, and will provide tools to study the processes underlying many biological phenomena in living systems.

\section{Acknowledgments}

I would like to thank Dr. K. Kikuchi, Dr. Y. Urano, Dr. H. Kojima and many graduate students as collaborators in Department of Chemistry and Biology, Graduate School of Pharmaceutical Sciences, the University of Tokyo for experimental assistance and fruitful discussions. The work was supported in part by research grants from the Ministry 
of Education, Culture, Sports, Science and Technology of Japanese Government.

\section{References}

[1] Palmer, R.M.J., Ferrige, A.G., and Moncada, S.: Nitric oxide release accounts for the biological activity of endotheliumderived relaxing factor. Nature, 327, 524-526, 1987.

[2] Nagano, T., Takizawa, H., and Hirobe, M.: Reaction of nitric oxide with amines in the presence of dioxygen. Tetrahedron Lett., 36, 8239-8242, 1995.

[3] Kojima, H., Nakatsubo, N., Kikuchi, K., Kawahara, S., Kirino, Y., Nagoshi, H., Hirata, Y., and Nagano, T.: Detection and imaging of nitric oxide with novel fluorescent indicators: diaminofluoresceins. Anal. Chem., 70, 2446-2453, 1998.

[4] Nakatsubo, N., Kojima, H., Kikuchi, K., Nagoshi, H., Hirata, Y., Maeda, D., Imai, Y., Irimura, T., and Nagano, T.: Direct evidence of nitric oxide production from bovine aortic endothelial cells using with new fluorescence indicators: diaminofluoresceins. FEBS Lett., 427, 263-266, 1998.

[5] Kojima, H., Sakurai, K., Kikuchi, K., Kawahara, S., Kirino, Y., Nagashi, H., Hirata, Y., and Nagano, T.: Development of a fluorescent indicator for nitric oxide based on the fluorescein chromophore. Biol. Pharm. Bull., 46, 373-375, 1998.

[6] Kojima, H., Urano, Y., Kikuchi, K., Higuchi, T., and Nagano, T.: Fluorescent indicators for imaging nitric oxide production. Angew. Chem. Int. Ed. Engl., 38, 3209-3212, 1999.

[7] Kojima, H., Hirata, M., Kudo, Y., Kikuchi, K., and Nagano, T.: Visualization of oxygen concentration-dependent production of nitric oxide in rat hippocampal slices during aglycemia. J. Neurochem., 76, 1404-1410, 2001.

[8] Kojima, H., Hirotani, M., Nakatsubo, N., Kikuchi, K., Urano, Y., Higuchi, T., Hirata, Y., and Nagano, T.: Bioimaging of nitric oxide with fluorescent indicators based on rhodamine chromophore. Anal. Chem., 73, 1967-1973, 2001.

[9] Izumi, S., Urano, Y., Hanaoka, K., Terai, T., and Nagano, T.: A simple and effective strategy to increase the sensitivity of fluorescence probes in living cells. J. Am. Chem. Soc., in press.

[10] Gabe, Y., Urano, Y., Kikuchi, K., Kojima, H., and Nagano, T.: Highly sensitive fluorescence probes for nitric oxide based on boron dipyrromethane chromophore-rational design of potentially useful bioimaging fluorescence probe. $\mathrm{J}$. Am.
Chem. Soc., 126, 3357-3367, 2004.

[11] Sasaki, E., Kojima, H., Nishimatsu, H., Urano, Y., Kikuchi, K., Hirata, Y., and Nagano, T.: Highly sensitive near-infrared fluorescence probes for nitric oxide and their application to isolated organs. J. Am. Chem. Soc., 127, 3684-3685, 2005.

[12] Ueno, T., Urano, Y., Setsukinai, K., Takakusa, H., Kojima, H., Kikuchi, K., Ohkubo, K., Fukuzumi, S., and Nagano, T.: Rational principles for modulating fluorescence properties of fluorescein. J. Am. Chem. Soc., 126, 14079-14085, 2004.

[13] Ueno, T., Urano, Y., Kojima, H., and Nagano, T.: Mechanism-based molecular design of highly selective fluorescence probes for nitrative stress. J. Am. Chem. Soc., 128, 10640-10641, 2006.

[14] Hempel, S.L., Buettner, G.R., O’Malley, Y.Q., Wessels, D.A., and Flaherty, D.M.: Dihydrofluorescein diacetate is superior for detecting intracellular oxidants: Comparison with $2^{\prime}, 7^{\prime}-$ dichlorodihydrofluorescein diacetate, 5(and 6)-carboxy-2',7'dichlorodihydrofluorescein diacetate, and dihydrorhodamine 123. Free Rad. Biol. Med., 27, 146-159, 1999.

[15] Setsukinai, K., Urano, Y., Kakinuma, K., Majima, M.J., and Nagano, T.: Development of novel fluorescence probes that can reliably detect reactive oxygen species and distinguish specific species. J. Biol. Chem., 278, 3170-3175, 2003.

[16] Koide, Y., Urano, Y., Kemmoku, S., Kojima, H., and Nagano, T.: Design and synthesis of fluorescent probes for selective detection of highly reactive oxygen species in mitochondria of living cells. J. Am. Chem. Soc., 129, 10324-10325, 2007.

[17] Umezawa, N., Tanaka, K., Urano, Y., Kikuchi, K., Higuchi, T., and Nagano, T.: Novel fluorescent probes for singlet oxygen. Angew. Chem. Int. Ed. Engl., 38, 2899-2901, 1999.

[18] Tanaka, K., Miura, T., Umezawa, N., Urano, Y., Kikuchi, K., Higuchi, T., and Nagano, T.: Rational design of fluoresceinbased fluorescence probes. Mechanism-based design of a maximum fluorescence probe for singlet oxygen. J. Am. Chem. Soc., 123, 2530-2536, 2001.

[19] Urano, Y., Kamiya, M., Kanda, K., Ueno, T., Hirose, K., and Nagano, T.: Evoluation of fluorescein as a platform for finely tunable fluorescence probes. J. Am. Chem. Soc., 127, 48884894, 2005.

[20] Kenmoku, S., Urano, Y., Kojima, H., and Nagano, T.: Development of a highly specific, rhodamine-based fluorescence probe for hypochlorous acid and its application to realtime imaging of phagocytosis. J. Am. Chem. Soc., 129, 73137318, 2007. 